\title{
The radio structure of radio-quiet quasars
}

\author{
C. Leipski ${ }^{1}$, H. Falcke ${ }^{2,3}$, N. Bennert ${ }^{1,4}$, and S. Hüttemeister ${ }^{1}$ \\ 1 AIRUB, University of Bochum, Universitätsstrasse 150, 44780 Bochum, Germany \\ e-mail: [leipski;nbennert; huette] @astro.rub.de \\ 2 Radio Observatory Westerbork, ASTRON, PO Box 2, 7990 AA Dwingeloo, The Netherlands \\ e-mail: falcke@astron.nl \\ 3 Department of Astrophysics, Radboud University, 6525 ED Nijmegen, The Netherlands \\ 4 Institute of Geophysics and Planetary Physics, University of California, Riverside, CA 92521, USA \\ e-mail: nicola.bennert@ucr . edu
}

Received 5 October 2005 / Accepted 26 April 2006

\begin{abstract}
Aims. We investigate the radio emitting structures of radio-quiet active galactic nuclei with an emphasis on radio-quiet quasars to study their connection to Seyfert galaxies.

Methods. We present and analyse high-sensitivity VLA radio continuum images of 14 radio-quiet quasars and six Seyfert galaxies. Results. Many of the low redshift radio-quiet quasars show radio structures that can be interpreted as jet-like outflows. However, the detection rate of extended radio structures on arcsecond scales among our sample decreases with increasing redshift and luminosity, most likely due to a lack of resolution. The morphologies of the detected radio emission indicate strong interactions of the jets with the surrounding medium. We also compare the radio data of seven quasars with corresponding HST images of the [O III] emitting narrowline region (NLR). We find that the scenario of interaction between the radio jet and the NLR gas is confirmed in two sources by structures in the NLR gas distribution as previously known for Seyfert galaxies. The extended radio structures of radio-quiet quasars at sub-arcsecond resolution are by no means different from that of Seyferts. Among the luminosities studied here, the morphological features found are similar in both types of objects while the overall size of the radio structures increases with luminosity. This supports the picture where radio-quiet quasars are the scaled-up versions of Seyfert galaxies. In addition to known luminosity relations we find a correlation of the NLR size and the radio size shared by quasars and Seyferts.
\end{abstract}

Key words. galaxies: active - galaxies: jets - galaxies: Seyfert

\section{Introduction}

The population of active galactic nuclei (AGN) shows a well known wide spread in radio luminosity (Miller et al. 1990; Kellermann et al. 1989, 1994; Miller et al. 1993; Kukula et al. 1998; Xu et al. 1999). Introducing the $R$ parameter as the ratio of radio flux at $5 \mathrm{GHz}$ and optical flux at $4400 \AA$ (Kellermann et al. 1989; Falcke et al. 1996b), the radio-loud objects are separated from the radio-quiet objects: usually, the radio-loud quasars have $R>100$ and $5 \mathrm{GHz}$ luminosities greater than $10^{26} \mathrm{~W} \mathrm{~Hz}^{-1}$. The radio-quiet ones typically have $R$ values between 0.1 and 10 and luminosities less than $10^{24} \mathrm{~W} \mathrm{~Hz}^{-1}$ at $5 \mathrm{GHz}$. Thus, the separation between the classes is nearly three orders of magnitude in radio luminosity (Kellermann et al. 1989, 1994). Radio-loud quasars (RLQs) and radio-galaxies are usually dominated by prominent double-lobe structures extending to tenth or hundreds of kpc. These extended structures in radio-loud objects are also dominating in luminosity by outshining the emission from the active central region by factors of 10 to 100 , resulting in a very low core-to-lobe flux ratio. On the other hand, radio-quiet quasars (RQQs) only show small scale structures of typically a few kpc with most of the flux concentrated in the nuclear region (e.g. Miller et al. 1990; Kellermann et al. 1994). Nevertheless, some RQQs are known to show extended structures reminiscent of radio-loud objects, but on very low flux-density scales
(Kellermann et al. 1994; Blundell \& Rawlings 2001). However, systematic high-dynamic range radio imaging studies of RQQs are still missing.

At lower (radio) luminosities, Seyfert galaxies also show compact structures, often dominated by a central unresolved component, sometimes accompanied by jet-like features (Ulvestad et al. 1981; Kukula et al. 1995; Schmitt et al. 2001; Ho \& Ulvestad 2001). Comparing the snapshot data of Seyfert galaxies and RQQs, there is no significant difference in the radio structure in both types of radio-quiet AGN.

However, in high sensitivity data, the diversity in radio structure of Seyfert galaxies is obvious (Ulvestad \& Wilson 1989; Capetti et al. 1996; Falcke et al. 1998). The morphologies can comprise linearly arranged multiple components (interpreted as emission knots in a jet), one- or two-sided diffuse jets or complicated structures that are, nevertheless, often well collimated. These radio structures coincide in shape and/or direction with the line-emitting gas of the narrow-line region (NLR), indicating that the radio ejecta are intimately connected to the NLR gas (Falcke et al. 1998).

In the ongoing debate of the evolution of active galaxies with luminosity, these findings of structured NLRs in Seyfert galaxies lead Bennert et al. (2002) to investigate the NLR structure of RQQs with high-resolution. They find resolved and structured quasar NLRs that closely resemble that of Seyfert 
Table 1. VLA observation Log.

\begin{tabular}{|c|c|c|c|c|c|c|c|}
\hline & \multirow[b]{2}{*}{$\begin{array}{l}\text { Object } \\
\text { (1) }\end{array}$} & \multicolumn{2}{|c|}{ Optical position } & \multirow[b]{2}{*}{$\begin{array}{l}z_{\text {hel }} \\
(4)\end{array}$} & \multirow[b]{2}{*}{$\begin{array}{c}R \\
(5)\end{array}$} & \multirow{2}{*}{$\begin{array}{c}\text { Frequency } \\
\text { (GHz) } \\
\text { (6) }\end{array}$} & \multirow{2}{*}{$\begin{array}{l}\text { On-source } \\
\text { time } \\
(7)\end{array}$} \\
\hline & & $\begin{array}{c}\alpha_{2000} \\
(2)\end{array}$ & $\begin{array}{c}\delta_{2000} \\
(3)\end{array}$ & & & & \\
\hline \multirow{7}{*}{ Quasars } & PG 0026+129 & 002913.73 & +131604.3 & 0.142 & 1.10 & 8.4 & $2: 02$ \\
\hline & PG $0052+251$ & 005452.16 & +252538.7 & 0.155 & 0.24 & 4.8 & $3: 57$ \\
\hline & PG $0157+001$ & 015950.21 & +00 2340.6 & 0.163 & 2.10 & 8.4 & $2: 22$ \\
\hline & PG $0953+414$ & 095652.50 & +411540.6 & 0.2341 & 0.44 & 4.8 & $3: 45$ \\
\hline & PG 1012+008 & 101454.88 & +00 3336.8 & 0.1874 & 0.50 & 8.4 & $2: 46$ \\
\hline & PG 1049-005 & 105151.50 & -005116.6 & 0.3599 & 0.25 & 4.8 & $3: 34$ \\
\hline & PG $1307+085$ & 130947.03 & +08 1949.3 & 0.155 & 0.10 & 4.8 & 3:07 \\
\hline \multirow{7}{*}{ Quasars } & PG 0003+199 & 000619.193 & +201210.51 & 0.0258 & 0.27 & 4.8 & $2: 59$ \\
\hline & PG $1119+120$ & 112147.102 & +114418.28 & 0.0502 & 0.15 & 4.8 & $2: 33$ \\
\hline & PG 1149-110 & 115203.505 & -112223.87 & 0.049 & 0.88 & 4.8 & 3:05 \\
\hline & PG $1351+640$ & 135315.847 & +634545.72 & 0.0882 & 4.30 & 4.8 & $3: 54$ \\
\hline & PG $1534+580$ & 153552.358 & +575409.17 & 0.0296 & 0.70 & 4.8 & $3: 47$ \\
\hline & PG $1612+261$ & 161413.214 & +260416.40 & 0.131 & 2.80 & 4.8 & $3: 17$ \\
\hline & PG 2130+099 & 213227.814 & +100819.49 & 0.063 & 0.32 & 4.8 & $2: 12$ \\
\hline \multirow{6}{*}{ Seyferts } & Mrk 612 & 033040.9 & -030816 & 0.0203 & & 8.4 & $1: 30$ \\
\hline & ESO 428-G14 & 071631.2 & -291928 & 0.0054 & & 8.4 & $1: 42+1: 05$ \\
\hline & NGC 2639 & 084338.1 & +501220 & 0.0111 & & 8.4 & 1:06 \\
\hline & NGC 2992 & 094542.0 & -141935 & 0.0077 & & 8.4 & 1:06 \\
\hline & Mrk 266 & 133817.5 & +481637 & 0.0279 & & 8.4 & $1: 39$ \\
\hline & NGC 5643 & 143240.8 & -441029 & 0.004 & & 8.4 & $1: 47+2: 19$ \\
\hline
\end{tabular}

Note: the first seven RQQs are also included in the HST sample of Bennert et al. (2002). (2)-(3) The optical position was taken from Miller et al. 1993 and calculated for J2000 (in ${ }^{\mathrm{h} \mathrm{min} \mathrm{s}}$ and ${ }^{\circ}{ }^{\prime \prime}$ ). (4) heliocentric redshift as provided by NED. (5) R parameter taken from Kellermann et al. (1994). (7) on-source integration time in hrs:min.

galaxies. Moreover they discovered a close correlation between the size and the luminosity of the NLR, connecting Seyfert galaxies and RQQs over a few orders of magnitude in luminosity. These results strongly support the idea that, amongst radioquiet AGN, RQQs are the high-power siblings of Seyfert galaxies. Since the close connection of Seyferts and RQQs is thus supported in the optical/NLR, we here explore the question if this can in fact be extended to the radio regime. We investigate if the interaction of radio ejecta and NLR gas, a typical phenomenon of active galaxies, persists with increasing luminosity. Therefore, we combine high-resolution, high-sensitivity radio and optical data for a sample of RQQs. We present radio continuum maps for 14 RQQs and some Seyfert galaxies to analyse the similarities and differences in both types of objects.

In Sects. 2 and 3, observations, data reduction, and data analysis are summarised. In Sect. 4, we present the results on an object-by-object basis. In Sect. 5, we discuss the results and their implications for the role of radio jets. We close with the concluding remarks in Sect. 6.

\section{Observations and data reduction}

This paper makes use of four different samples that we now present briefly: first, the quasar sample of Bennert et al. (2002). The objects are the seven most-luminous (in [O III]) quasars with $z<0.5$ in the Palomar Green (PG) quasar sample (Schmidt \& Green 1983). Only for $z<0.5$ the PG quasar sample is complete and not affected by a selection bias (e.g. Wampler \& Ponz 1985). Only for this sub-sample HST, emission-line images are available.

The second sample consists of seven PG quasars with redshift $z<0.2$ and [O III] emission line fluxes $>5 \times$ $10^{-14} \mathrm{erg} \mathrm{s}^{-1} \mathrm{~cm}^{-2}$. This corresponds to [O III] luminosities exceeding those for typical Seyfert galaxies, thereby filling the gap between quasars and Seyferts in the NLR size-luminosity correlation of Bennert et al. (2002). These objects were selected as "transition objects" in terms of their [O III] luminosities and (expected) NLR sizes and are thus of special interest.

Third, a sample of six Seyfert galaxies that were selected on the basis of their extended morphological appearance in radio snapshot surveys. This sample is presented in the appendix.

For all these objects, the basic parameters of the observations are given in Table 1. In Sect. 5, we will make use of the Seyfert sample of Kinney et al. (2000) and Schmitt et al. (2001, 2003). These objects were selected on the basis of a mostly isotropic property, their $60 \mu \mathrm{m}$ fluxes and their warm infrared colours.

The data for the first three samples were newly obtained using the Very Large Array (VLA ${ }^{1}$; Thompson et al. 1980) in the $\mathrm{A}, \mathrm{BnA}$, and $\mathrm{B}$ configuration at $4.8 \mathrm{GHz}$ or $8.4 \mathrm{GHz}$ with two intermediate frequencies (IFs) of $50 \mathrm{MHz}$ bandwidth. The details of the observations are given in Table 1 . The data reduction was performed in the typical manner using the AIPS software. All bright sources were subject to several iterations of self-calibration. Natural- and uniform-weighted (with a robust parameter of -4) maps were produced with various cleaning depths, taperings and CLEAN gain factors.

In Sect. 4 we present and discuss the radio maps of the newly observed objects on an object-by-object basis. The maps of the quasars show compact cores, often accompanied by weak extended emission or additional unresolved components. All maps in this paper are shown in contours with spacings of $\sqrt{2}^{n}$, starting at $3 \sigma_{\text {rms }}$ as given in Table 2.

\section{Data analysis}

Peak positions of the radio sources were determined in the high resolution uniform-weighted maps by fitting a two-dimensional GAUSS-function to the central peak using the AIPS-task IMFIT.

1 The National Radio Astronomy Observatory is a facility of the National Science Foundation operated under cooperative agreement by Associated Universities, Inc. 
Table 2. Source properties.

\begin{tabular}{|c|c|c|c|c|c|c|c|c|c|c|}
\hline \multirow[b]{2}{*}{$\begin{array}{l}\text { Object } \\
\text { (1) }\end{array}$} & \multicolumn{2}{|c|}{ Peak position } & \multirow{2}{*}{$\begin{array}{c}\text { Peak flux } \\
\text { mJy } \\
(4)\end{array}$} & \multirow{2}{*}{$\begin{array}{c}\text { Total flux } \\
\text { mJy } \\
(5)\end{array}$} & \multirow{2}{*}{$\begin{array}{l}\text { Size } \\
\text { in " } \\
(6)\end{array}$} & \multirow{2}{*}{$\begin{array}{c}3 \sigma_{\text {rms }} \\
10^{-5} \mathrm{Jy} \\
(7)\end{array}$} & \multirow{2}{*}{$\begin{array}{c}\text { Total flux } \mathrm{B} \\
\text { mJy } \\
(8)\end{array}$} & \multirow{2}{*}{\multicolumn{2}{|c|}{$\begin{array}{c}\text { Luminosity } \\
10^{21} \frac{\mathrm{W}}{\mathrm{Hz}}\end{array}$}} & \multirow{2}{*}{$\begin{array}{c}\text { Scale } \\
\text { pc/" } \\
(11)\end{array}$} \\
\hline & $\begin{array}{c}\alpha_{2000} \\
\text { (2) }\end{array}$ & $\begin{array}{c}\delta_{2000} \\
\text { (3) }\end{array}$ & & & & & & & & \\
\hline PG 0026+129 & 002913.700 & +131604.00 & 0.17 & 0.28 & 0.6 & 4.5 & 2.37 & 14.52 & 129.95 & 2442.6 \\
\hline PG $0052+251$ & 005452.116 & +252539.03 & 0.58 & 0.61 & $<0.09$ & 3 & - & 38.45 & & 2631.8 \\
\hline PG $0157+001$ & 015950.252 & +002340.89 & 2.98 & 4.23 & 2.6 & 3 & 5.55 & 298.74 & 391.96 & 2746.7 \\
\hline PG 0953+414 & 095652.400 & +411522.16 & 0.13 & 0.18 & 1.3 & 3 & 0.18 & 29.16 & 29.16 & 3684.0 \\
\hline PG $1012+008$ & 101454.900 & +00 3337.46 & 0.10 & 0.25 & 1.0 & 3 & 0.65 & 24.72 & 64.28 & 3106.6 \\
\hline PG 1049-005 & 105151.446 & -005117.70 & 0.22 & 0.25 & $<0.27$ & 3 & - & 109.57 & & 4987.8 \\
\hline PG $1307+085$ & 130947.000 & +08 1948.16 & 0.13 & 0.15 & 1.1 & 3 & - & 9.74 & & 2661.6 \\
\hline PG 0003+199 & 000619.537 & +201210.61 & 3.49 & 3.58 & $<0.08$ & 5 & - & 4.69 & & 481.4 \\
\hline PG $1119+120$ & 112147.122 & +114418.93 & 0.08 & 0.39 & 5.6 & 3 & 1.04 & 2.38 & 6.34 & 985.7 \\
\hline PG $1149-110$ & 115203.549 & -112224.10 & 1.08 & 1.94 & 2.4 & 5.5 & - & 11.28 & & 964.8 \\
\hline PG $1351+640$ & 135315.828 & +634545.52 & 7.31 & 8.48 & 3.5 & 3 & - & 161.70 & & 1626.8 \\
\hline PG $1534+580$ & 153552.400 & +575409.53 & 2.00 & 2.21 & $<0.22$ & 5 & - & 4.36 & & 584.8 \\
\hline PG $1612+261$ & 161413.221 & +260416.27 & 1.19 & 5.27 & 2.6 & 5.5 & 6.12 & 234.28 & 272.07 & 2299.8 \\
\hline PG 2130+099 & 213227.816 & +1008 19.25 & 0.68 & 2.41 & 2.9 & 6 & - & 21.61 & & 1172.3 \\
\hline Mrk 612 & 033040.906 & $\begin{array}{llll}-03 & 08 & 14.56\end{array}$ & 0.19 & 0.68 & 1.9 & 3 & - & 0.59 & & 393.8 \\
\hline ESO 428-G14 & 071631.165 & -291928.37 & 3.84 & 18.95 & 4.8 & 4 & - & 1.50 & & 122.6 \\
\hline NGC 2639 & 084338.078 & +501219.98 & 132.16 & 152.97 & 2.0 & 7 & - & 44.80 & & 233.4 \\
\hline NGC 2992 & 094541.946 & -141934.67 & 4.81 & 12.00 & 7.6 & 4.5 & - & 2.03 & & 178.3 \\
\hline Mrk 266 & 133817.786 & +481641.16 & 3.47 & 15.00 & 12.4 & 5 & - & 27.06 & & 560.5 \\
\hline NGC 5643 & 143240.703 & -441027.41 & 5.68 & 11.70 & 41.0 & 4 & - & 0.55 & & 94.7 \\
\hline
\end{tabular}

Note: (2)-(3) in ${ }^{\mathrm{h} \min \mathrm{s}}$ and ${ }^{\circ \prime \prime}$. (6) major extension (diameter) of source emission, a "<" means that only an upper limit can be estimated. (8) B-Array flux. (9)-(10) luminosity from the A and B-Array fluxes, respectively. (4)-(10) flux, size, $3 \sigma_{\text {rms }}$, and luminosity at the observed frequency shown in Table 1.

For diffuse and extended sources for which a multi-component GAUSSIAN-fit is not suited to represent the source structure the total flux was derived by summing the flux inside the $3 \sigma_{\text {rms }}$ contour. The noise in the map was determined over large areas near the source using the task TVSTAT.

The diameter of the radio sources was determined by measuring the widest extent of the source in the $3 \sigma_{\text {rms }}$ contour. This diameter was corrected for the elongation of the beam. However, this correction has only a minor impact on the overall source size and becomes less important with increasing total extent of the source. For the compact sources, the deconvolution of the beam shape and the apparent source shape was performed by the task IMFIT. For all cases in which a source appeared compact, it was only marginally resolved after deconvolution. We take this marginal extent only as an upper limit on the source size.

From the fluxes and diameters, luminosities and linear distances were calculated using a homogeneous flat world model which includes Einstein's cosmological constant $\Lambda$ in agreement with the recent results of the Wilkinson Microwave Anisotropy Probe (Bennet et al. 2003: $H_{0}=71 \mathrm{~km} \mathrm{~s}^{-1}, \Omega_{\text {matter }}=0.27$ and $\Omega_{\Lambda}=0.73$ ). Results from the data analysis are given in Table 2 .

\section{Results}

\subsection{Radio-quiet quasars}

The radio-quiet quasars presented here consist of two groups, each with seven objects, and all taken from the PG quasar sample (Schmidt \& Green 1983). The seven objects in the first part of Tables 1 and 2 have been imaged with HST in the redshifted [O III] line by Bennert et al. (2002).

Their emission-line images and our new radio maps are shown below. Table 2 lists the central coordinates, integrated and peak fluxes, as well as sizes and noise levels in the maps. Derived luminosities and linear source scales are also given.

\subsubsection{Seven RQQs with HST NLR images}

PG 0026+129. This source shows no remarkable extent in neither the HST nor the high-resolution VLA image (Fig. 1). Kellermann et al. (1989) found that only $4 \%$ of the total flux is concentrated in the core of the object (at $4.8 \mathrm{GHz}$ ) but they did not find any extended structures. Our A-Array radio map does not show any extent either, not even on a tapered map. However, on an additional map taken with the VLA in the B-configuration, we detect diffuse emission with an extent of nearly $20 \mathrm{kpc}$ that was totally missed by our A-Array observations. Only $12 \%$ of the B-Array flux was previously detected with the A-Array. Interestingly, the slight elongation on the A-Array images is roughly east-west, but the B-Array image is elongated north-south.

PG 0052+251. For this source, Bennert et al. (2002) reported an extended structure in their HST [O III] emission-line map. This image is reproduced as a contour plot in Fig. 2. The corresponding radio map is also shown, but there is no elongation in the direction of the optical line emission component. The extension to the south-west on a $1.4 \mathrm{GHz}$ map mentioned by Kukula et al. (1998) in agreement with the optical image is not confirmed in our deep 4.8 GHz map. As Kukula et al. (1998) noted, this structure is possibly not real. Thus, there is no correlation between [O III] emission and radio emission for this separated knot.

PG 0157+001. This triple radio source is clearly related to the emission-line structures shown by Bennert et al. (2002) (Fig. 3). The western knot of radio emission seems to represent the termination point of a jet, which is stopped in the interstellar medium. This is strengthened by a bow-shock like structure of swept-up material in the emission-line image which is very similar to what is seen in Mrk 573 (Pogge \& de Robertis 1995; Falcke et al. 1998; Ferruit et al. 1999). As for Mrk 573, the interaction of the radio jet with the NLR gas producing the 

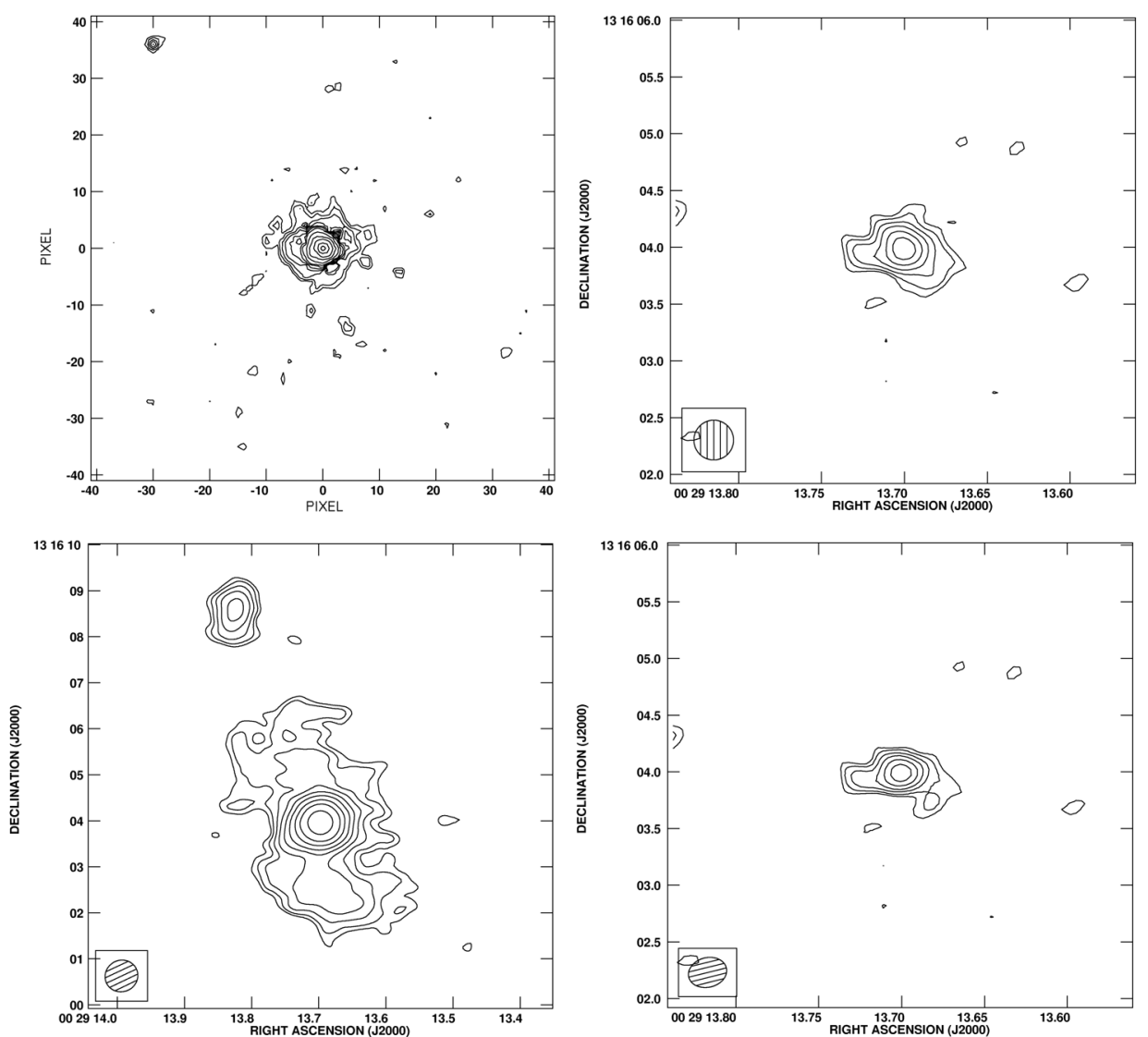

Fig. 1. HST and VLA images of PG 0026+129. Images are $4 " \times 4 "$ wide, if not stated otherwise. Upper left: HST linear ramp filter (LRF) image in the [O III] line. Pixel offsets from centre correspond to $0.05^{\prime \prime} / \mathrm{px}$. Upper right: natural-weighted VLA A-Array map at $8.4 \mathrm{GHz}$ with a 0 '. $35 \mathrm{beam}$. Lower left: natural-weighted VLA B-Array map at $8.4 \mathrm{GHz}$ with a $500 \mathrm{k} \lambda$ Gaussian taper (beamsize $0{ }^{\prime} \cdot 72 \times 00^{\prime} 68$ ). The size of the image is $10^{\prime \prime}$. Lower right: natural-weighted VLA A-Array map at $8.4 \mathrm{Ghz}$ (beamsize 0 '.34 × 0.'26).
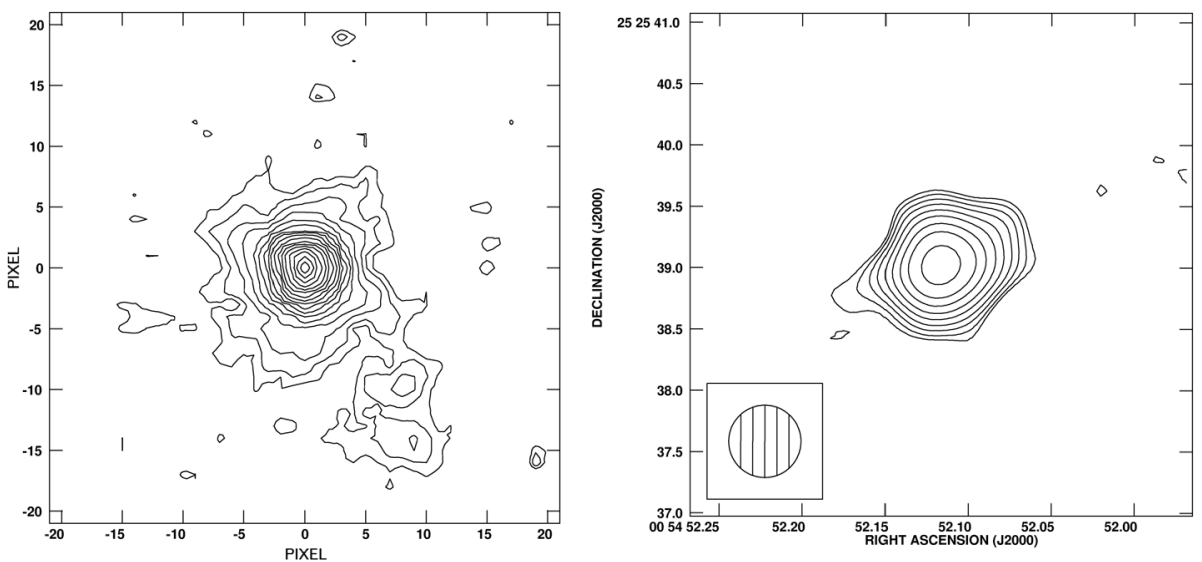

Fig. 2. HST and VLA images of PG 0052+251. Both images are $4 " \times 4^{\prime \prime}$ wide. Left: HST LRF image in the [O III] line. Pixel offsets from centre correspond to $0.1^{\prime \prime} /$ px. Right: natural-weighted VLA A-Array map at $4.8 \mathrm{GHz}$ with a beam of 0'.59.

bow-shock is confirmed by optical spectroscopy (Leipski \& Bennert 2006). There is no corresponding association of the optical emission line structures at the position of the eastern radio knot. In fact, the emission line gas is distributed to the south of this knot.

The eastern radio knot was missed by the snapshot images presented by Kellermann et al. (1989). The overall structure of the triple radio source resembles that of the large scale [O III] emission shown by Stockton \& MacKenty (1987). A VLA B-Array map shows no additional components in this source.
PG 0953+414. This quasar is elongated in the east-west direction in the radio regime (Fig. 4, right). In the corresponding [O III] emission-line image there is no significant extent, especially not in the direction of the radio extension (Fig. 4, left). The radio structure seen with the A-Array does not appear on a B-Array map where the source is unresolved at $4.8 \mathrm{GHz}$. Thus, a radio jet seems to be directed to the east-west causing extended but collimated radio emission on scales of a few kpc. This source was not detected by Kellermann et al. (1989).

PG 1012+008. This quasar is part of an interacting system (Bahcall et al. 1997). The interstellar medium is perturbed and it 

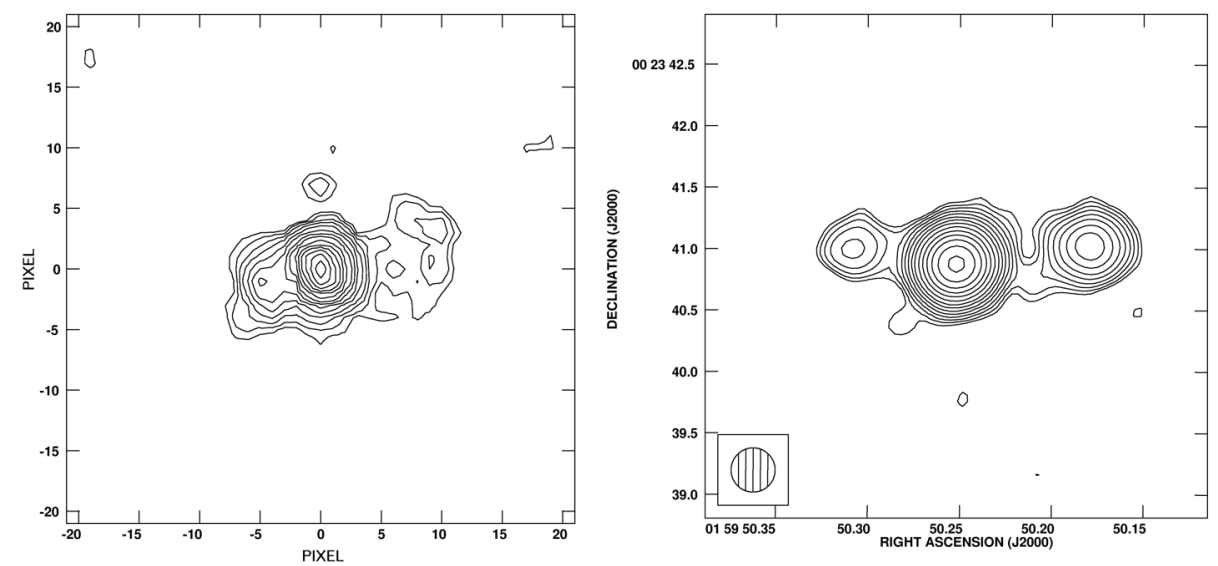

Fig. 3. Same as in Fig. 2 for PG $0157+001$. The VLA map is at $8.4 \mathrm{GHz}$ with a 0 ' 36 beam.
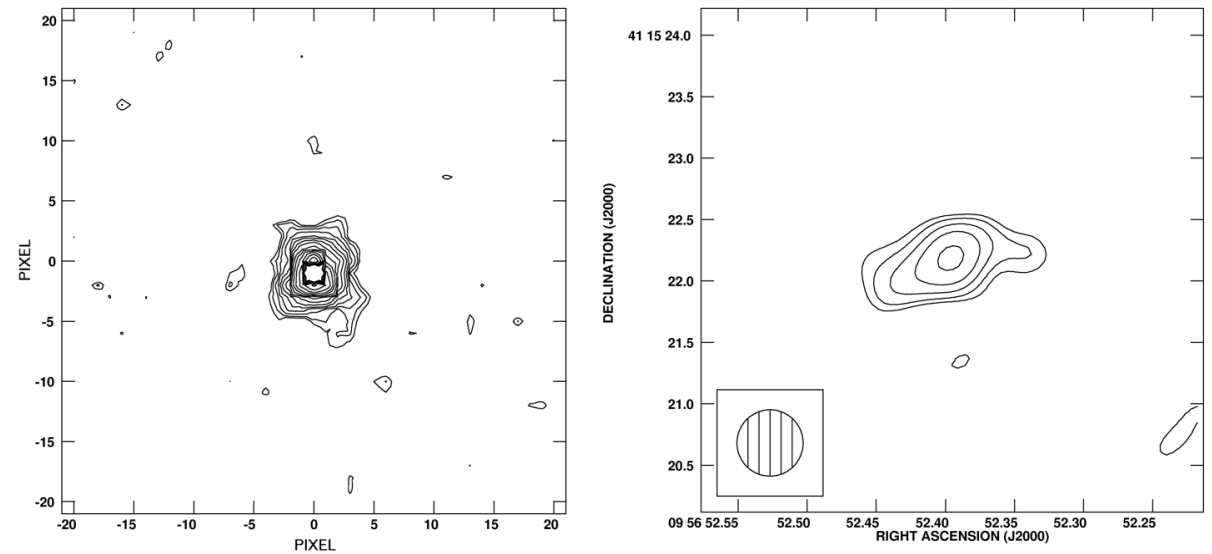

Fig. 4. Same as in Fig. 2 for PG $0953+414$. The VLA map is at $4.8 \mathrm{GHz}$ with a 0 '.5 beam.
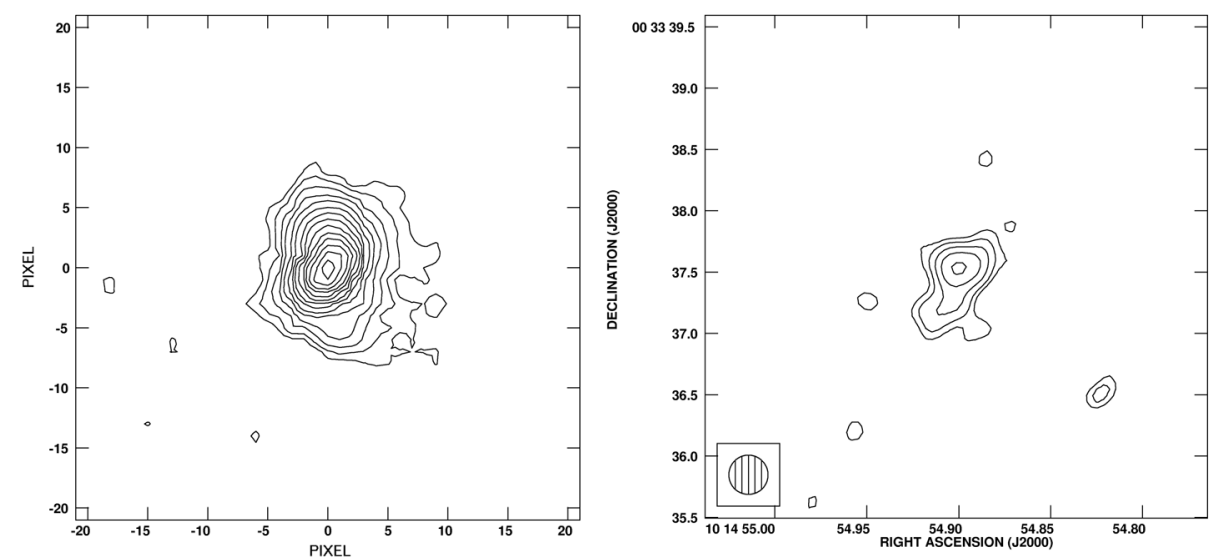

Fig. 5. Same as in Fig. 2 for PG 1012+008. The VLA map is at $8.4 \mathrm{GHz}$ with a 0.32 beam.

is difficult to say whether the structures are caused by the merger or by the radio-jet itself. The radio source consists of a nuclear source with a jet to the south (see Fig. 2, right). The jet seems to bend to the east as indicated by comparing the inner and outer isophotes. The same "bended" structure is apparent in the optical image where the inner isophotes display the shape of the radio source (Fig. 2, left). This indicates that the radio jet and the NLR gas are intimately connected. However, it is not clear what effect the galaxy interaction has on the apparent structures, especially on the "bending".

A corresponding B-Array map resolves no prominent additional structures, except one possible short extension to the north-west in the opposite direction of the jet detected in the A-Array.

PG 1049-005. This source appears pointlike at both wavelengths (Fig. 6), but the HST image is resolved due to the better resolution (Bennert et al. 2002). For the radio extent we can only give an upper limit. Note that the elongations in the HST image (Fig. 6, left) to the north-east and south-west and the minor elongations to the south-east and north-west are due to the secondary mirror supporting structure and, therefore, are artificial.

PG 1307+085. At both (radio and optical) wavelengths, a compact component can be seen as well as an elongation to the south-west (Fig. 7). While the radio map displays a secondary 

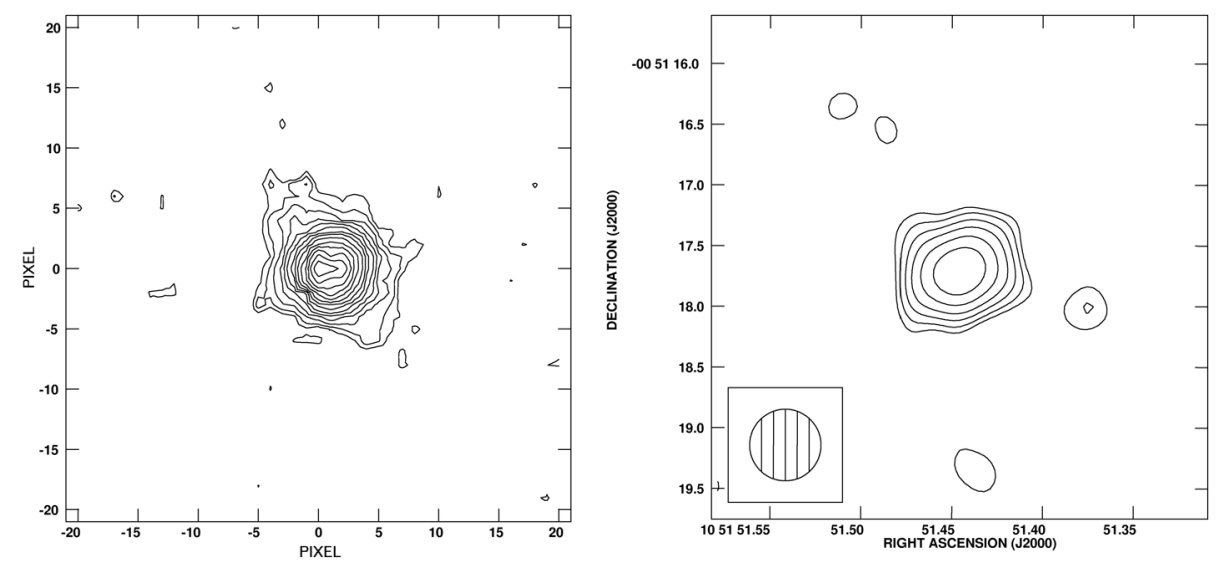

Fig. 6. Same as in Fig. 2 for PG 1049-005. The VLA map is at $4.8 \mathrm{GHz}$ with a 0.'59 beam.
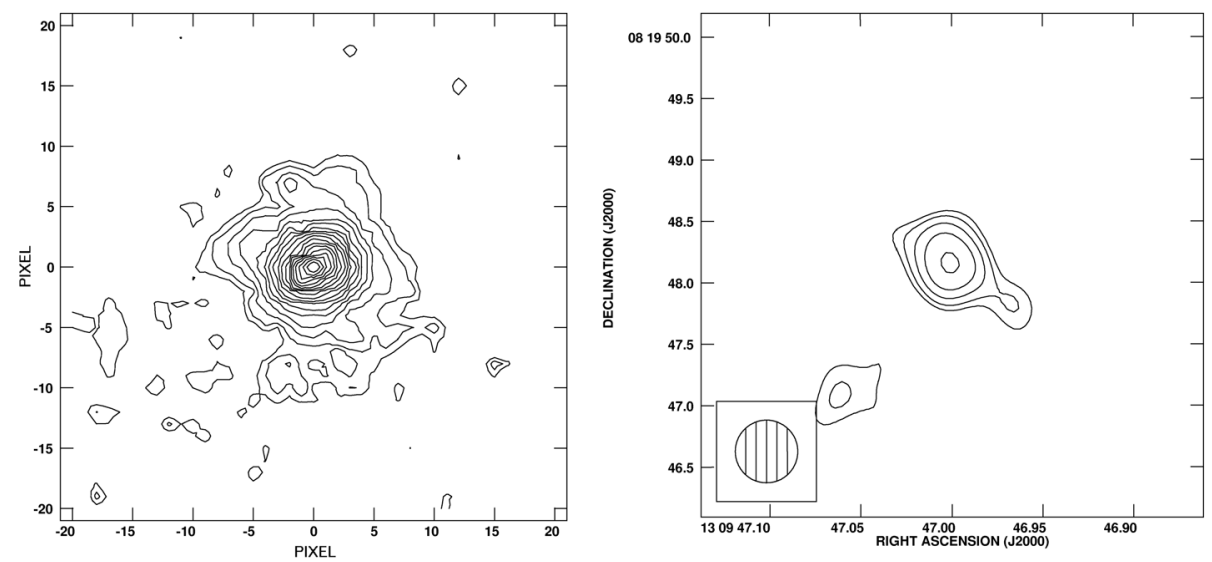

Fig. 7. Same as in Fig. 2 for PG $1307+085$. The VLA map is at $4.8 \mathrm{GHz}$ with a 0 '.51 beam.

component in the south-west that possibly is part (or the termination point) of a radio jet, the HST image shows diffuse emission in the same region. While these results are consistent with a radio-NLR interaction, they are by no means conclusive.

\subsubsection{Seven RQQs without HST NLR images}

For the following seven objects (the middle part of Tables 1 and 2), no optical emission-line images are available. Their redshifts and luminosities lie in between the redshifts and luminosities of the RQQs mentioned above and the Seyfert galaxies. Thus, these seven objects are very interesting as they connect Seyferts and RQQs. If there is indeed a close connection between all radio-quiet AGN, it shall be reflected by the radio structures in these objects.

PG 0003+199. A compact, unresolved radio source at 4.8 GHz (Fig. 8, left).

PG 1119+120. Three different components are visible in this source: A compact component in the south-west and at the centre as well as a one-sided jet to the north-east (Fig. 9). This jet is nearly two arcseconds long before it sharply bends to the northwest. The whole structure is embedded in a more diffuse emission, especially to the north of the south-western blob (Fig. 9, right). Leipski \& Bennert (2006) have shown that extended [O III] emission exists in this source and that the emission-line ratios suggest an interaction of the radio jet with the NLR gas.

PG 1149-110. A triple radio source extending 1".5 in roughly east-west direction (Fig. 10, left). Although the western component seems to become steadily weaker it has a peak that can be seen with different contour spacings. Neither Kellermann et al. (1989) nor Kukula et al. (1998) were able to detect any components in addition to an unresolved core.

PG 1351+640. This source is known to be strongly variable (Barvainis \& Antonucci 1989) and has a VLBA core (Blundell \& Beasley 1998; Ulvestad et al. 2005). However, on arcsecond scales we detect three aligned components with surrounding diffuse emission (Fig. 10, right). Kellermann et al. (1989, 1994) have only securely detected the central component.

PG 1534+580. Unresolved like PG 0003+199 (Fig. 8, right).

PG 1612+261. An one-sided jet heads 1".5 towards the south-west (Fig. 11, left). As in the case of PG 1149-110, three peaks can be identified in this source with adequate contour spacings. While one peak lies very close in the north-east of the central peak, another peak appears at the tip of the jet. Nevertheless, this source holds its one-sidedness. The $R$ value of PG $1612+261$ is remarkably high $(2.81$, Kellermann et al. 1989 ) for a radio-quiet quasar and comparison with flux values given by Kellermann et al. (1989) indicates variability. On an additional B-Array map there is no further structure detectable and only $14 \%$ of the B-Array flux is missed by the A-Array observations.

PG 2130+099. Another aligned triple radio source, but with well separated and clearly detected components (Fig. 11, right). The overall extent is nearly 2 '.5, orientated north-west/southeast. Especially the component to the north-west displays a remarkable sub-structure which is elongated perpendicular to the jet-direction, possibly indicating a transverse shock. 

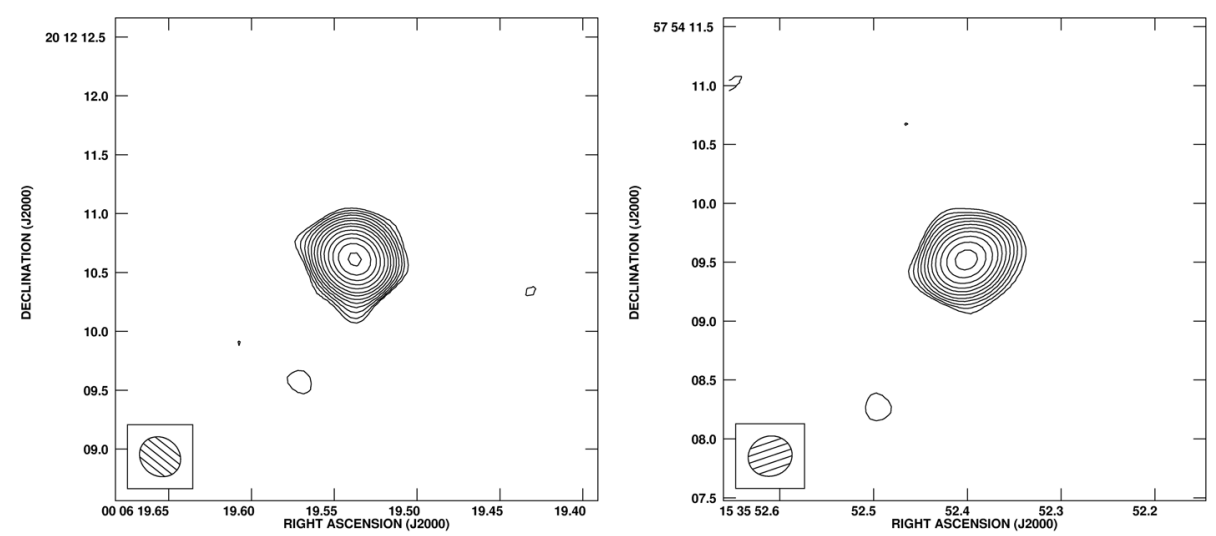

Fig. 8. VLA images of PG 0003+199 (left) and PG 1534+580 (right). Both images are 4" $\times 4$ " wide. Left: uniform-weighted VLA A-Array map at $4.8 \mathrm{GHz}$ with a $0.37 \times 0$ 0.32 beam. Right: uniform-weighted VLA A-Array map at $4.8 \mathrm{GHz}$ with a 0.'38 × 0.'34 beam.
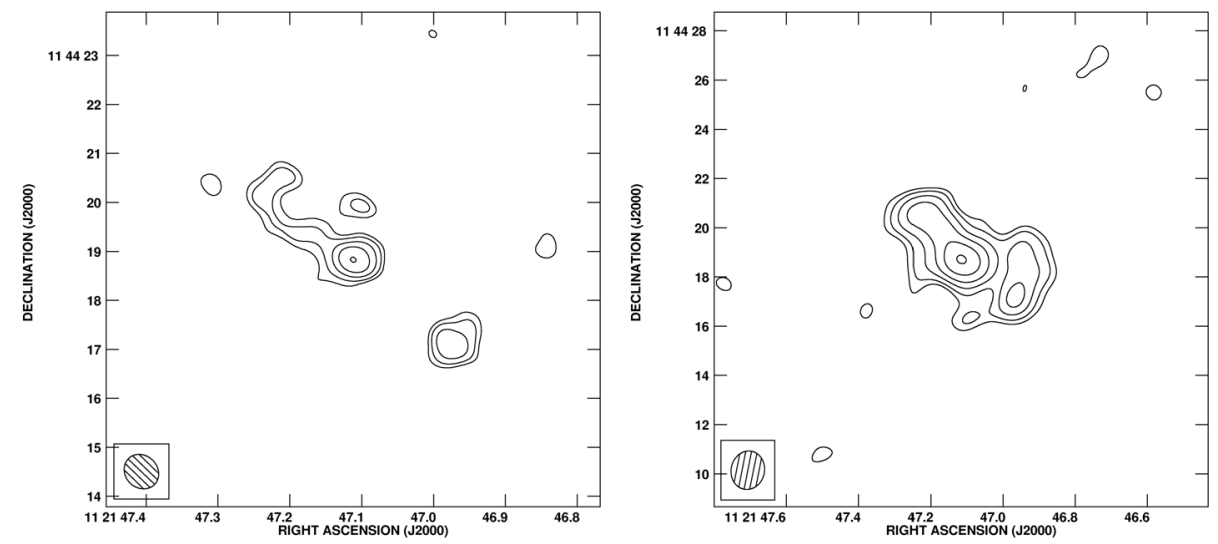

Fig. 9. VLA images of PG 1119+120. Left: $10^{\prime \prime} \times 10^{\prime \prime}$ wide image. Natural-weighted A-Array map at $4.8 \mathrm{GHz}$ with a UV taper $300 \mathrm{k} \lambda$ and a $0^{\prime} 75 \times 0$ ' 6 beam. Right: $20^{\prime \prime} \times 20^{\prime \prime}$ wide image. Natural-weighted VLA B-Array map at $4.8 \mathrm{GHz}$ with a $1^{\prime \prime} .6 \times 11^{\prime \prime} 4$ beam.

\section{Discussion}

\subsection{Structure of the radio emission}

\section{a) Sizes and luminosities}

To investigate the radio emission of radio-quiet quasars we first compare their sizes and luminosities with literature data before studying their morphologies in detail.

In luminosity, there is almost no overlap between the higher redshift RQQ sample and local $\left(v<5000 \mathrm{~km} \mathrm{~s}^{-1}\right)$ Seyfert samples (e.g. Morganti et al. 1999; Ulvestad \& Ho 2001; Ho \& Ulvestad 2001). However, the very bright end of the (rather powerful) $12 \mu \mathrm{m}+$ CfA sample extends well inside (but does not exceed) the luminosities of the higher redshift RQQs (Rush et al. 1996). On the other hand, the lower redshift RQQ sample connects the almost distinct local Seyfert and high- $z$ RQQ samples in terms of luminosity, but they hardly exceed the very brightest $12 \mu \mathrm{m}+$ CfA sources. Therefore, the higher redshift sample significantly increases the luminosity baseline for radio-quiet AGN, while the lower redshift sample aids to bridge Seyferts and quasars.

Considering the sizes, a very similar situation is achieved. But this time the size of even the largest RQQ in our study hardly exceeds the size of the largest Seyferts (e.g. Morganti et al. 1999; Ulvestad \& Ho 2001). However, the size of a RQQ with a given morphology is in fact larger than that of Seyferts of the same morphological type (see discussion below). Additionally, at higher luminosities the situation may be much more complex due to the possibility of AGN to harbour large scale low flux density emission (Fig. 1). Moreover, at the high luminosity end of our samples we start to enter the range in size and luminosity measured for the radio galaxies with the very lowest luminosities (de Ruiter et al. 1990; Neeser et al. 1995).

In the well known $L_{\text {radio }}-L_{[\mathrm{OIII}]}$ correlation for AGN (e.g. Miller et al. 1993; Falcke et al. 1995; Xu et al. 1999), the radioquiet population is clearly distinguished from the radio-loud population. While both populations share roughly the same range in $L_{[\mathrm{O} I I]}$, they are separated in $L_{\text {radio }}$ with only very little overlap. Given that $L_{[\mathrm{O} I I]}$ can be used as a measure for the total luminosity of the $\mathrm{AGN}^{2}$, some radio-quiet objects with the very highest (total) luminosities have sizes comparable to the radio-loud objects with the very lowest (total) luminosities ${ }^{3}$. However, in this apparent continuity the overlap between our radio-quiet objects and the radio-loud population in the radio regime is sparse and has to be addressed in detail elsewhere.

b) Detailed morphological comparison

The radio-quiet quasars observed here can be roughly divided into four groups on the basis of their radio morphology:

(i) quasars that are unresolved (or almost unresolved) and point-like (e.g. PG 0052+251, Fig. 2),

${ }^{2}$ Although this is widely accepted, recent results provide evidence that this simple picture might not be true (Haas et al. 2005).

3 This obviously reflects the distinction of radio-loud and radio-quiet objects via the R-parameter (e.g. Kellermann et al. 1989). 

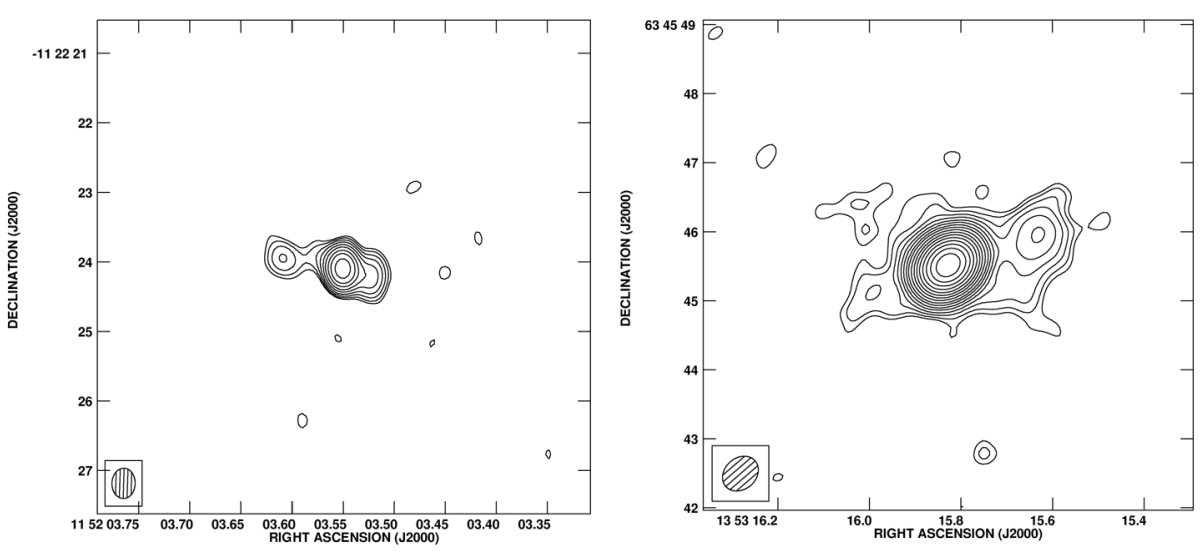

Fig. 10. VLA images of PG 1149-110 (left) and PG 1351+640 (right). Both images are 7" $\times 7 "$ wide. Left: uniform-weighted A-Array map at $4.8 \mathrm{GHz}$ with a 0! $44 \times 0$ 0'33 beam. Right: natural-weighted VLA A-Array map at $4.8 \mathrm{GHz}$ with a $0.56 \times 0.45$.
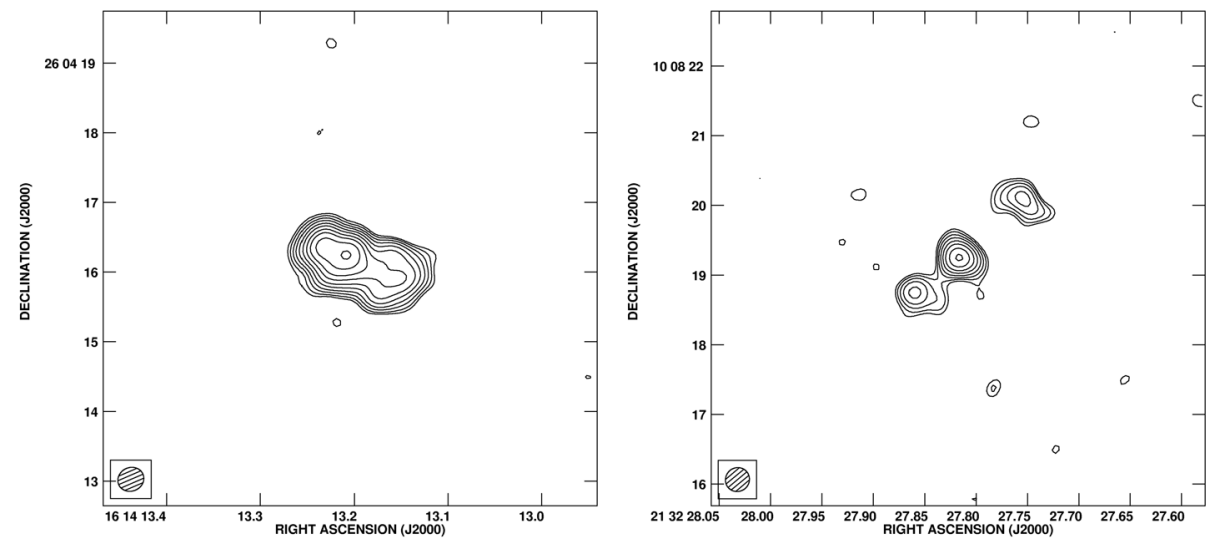

Fig. 11. VLA images of PG 1612+261 (left) and PG 2130+099 (right). All images are 7" $\times 7$ " wide. Left: uniform-weighted A-Array map at $4.8 \mathrm{GHz}$ with a $0^{\prime} .38 \times 00^{\prime} 34$ beam. Right: uniform-weighted VLA A-Array map at $4.8 \mathrm{GHz}$ with a 0.'35 × 0.'34 beam.

(ii) objects which exhibit symmetric, double-sided structures that steadily fade out (e.g. PG 0953+414, Fig. 4),

(iii) objects with clear sub-peaks (radio knots) on both side of the nucleus (e.g. PG 0157+001, Fig. 3),

(iv) quasars with strong asymmetry towards one side of the nucleus (e.g. PG 1119+120, Fig. 9).

All these types of radio structure can also be found in Seyfert galaxies (e.g. Ulvestad \& Wilson 1989; Kukula et al. 1995; Kinney et al. 2000; this paper). As the Seyfert galaxies presented here were selected on the basis of known extended radio emission (see Sect. 2 and Appendix A), we do not find counterparts to quasars in group (i). However, many Seyfert galaxies are known to be unresolved sources, even with sub-arcsecond resolution: Comparing the radio snapshot-surveys of Seyfert galaxies (e.g. Ulvestad et al. 1981; Schmitt et al. 2001) and quasars (e.g. Miller et al. 1993; Kellermann et al. 1994; Kukula et al. 1998), compact and unresolved objects are common to both object classes. But, studies using deep images with long integration times (e.g. Falcke et al. 1998; Blundell \& Rawlings 2001; this paper) have shown that sources appearing pointlike in snapshot surveys need not necessarily to be unresolved. Instead, low flux-density emission (e.g. PG 0026+129, Fig. 1, PG 1119+120, Fig. 9; Blundell \& Rawlings 2001) and additional components (e.g. PG $0157+001$, Fig. 3 ) could be detected - particularly when multi-array observations are used.

A prominent counterpart to group (ii) is the Seyfert galaxy NGC 5643 (Fig. A.3). This objects has similar structures as, for example, PG 0953+414 (Fig. 4). The much higher diversity in structure seen for NGC 5643 can be attributed to resolution effects. Calculating the linear scales of the radio emission, the structures of PG $0953+414$ turn out to be even larger $(\sim 5 \mathrm{kpc})$ than those in NGC $5643(\sim 4 \mathrm{kpc}$; Table 2$)$. PG $0026+129$ is thereby exceptional in this group as the overall structure is $\sim 20 \mathrm{kpc}$ large.

In group (iii), PG 0157+001 (Fig. 3) can be compared with, for example, Mrk 573. Both objects consist of multiple aligned (individually barely resolved) emitting knots in the radio regime. These radio knots are generally interpreted as the termination points of a jet that is stopped in the dense ISM where the (radio) emission is enhanced by compressed material and magnetic fields (e.g. Falcke et al. 1998). The optical emission-line gas thereby displays prominent bow-shock structures that are closely related to the radio knots indicating strong interaction (Falcke et al. 1998; Ferruit et al. 1999; Fig. 3). This interaction of a radio-jet that sweeps up material is confirmed spectroscopically for Mrk 573 (Ferruit et al. 1999) as well as for PG 0157+001 (Leipski \& Bennert 2006) by the presence of strong line-emitting components displaced in velocity. However, it should be noted that despite these similarities the linear distance of the interacting region from the nucleus is clearly larger in PG $0157+001(\sim 3300 \mathrm{pc})$ than in Mrk $573(\sim 700 \mathrm{pc})$.

The jet of PG $1119+120$ (Fig. 9) is comparable to the one in ESO 428-G14 (Fig. A.1), both being part of group (iv). The jets in these objects are pronounced only on one side of the nucleus and they stand out by their bended shape. As suggested by Falcke et al. (1998) for ESO 428-G14, the jet in PG 1119+120 is 
possibly hitting a dense cloud in the ISM that redirects it. In contrast to the scenario suggested for PG 0157+001 (and Mrk 573), the redirecting ISM cloud is too dense to produce a bow-shock (that should develop in a rather smooth distribution of surrounding material). Nevertheless, at the point where the redirection takes place, there is a hot spot or at least an "enhancement" in radio emission for both, PG 1119+120 (Fig. 9) and ESO 428-G14 (Fig. A.1). Again, the linear scales are clearly different as the redirection in ESO 428-G14 takes place at a distance of $\sim 500 \mathrm{pc}$ from the nucleus while this point is $\sim 2500 \mathrm{pc}$ away from the nucleus in PG $1119+120$. PG $1612+261$ can also be attributed to group (iv) with a jet asymmetry to the western side, though the jet is linear in this case.

Besides the mere morphological match, a certain radio structure seems to be accompanied by a similar distribution of the emission-line gas. For PG $0157+001$ and Mrk 573, there is radio and NLR emission on either side of the nucleus (Falcke et al. 1998, Fig. 3). Even if the NLR of NGC 5643 appears one-sided (Simpson et al. 1997) these authors suggest that the other side of the NLR is simply hidden by dust. In the framework of the structural match suggested here, this would be confirmed by the two-sided radio structure (Fig. A.3) as the radio emission is not affected by dust obscuration.

In contrast to the two-sided sources, objects with onesided jets (or at least a clear asymmetry to one side) show this asymmetry also in the NLR gas distribution, as found for PG 1012+008 and ESO 428-G14 (Fig. 5; and Falcke et al. 1998, respectively).

To summarise, there is no significant difference between the radio structures of Seyfert galaxies and radio-quiet quasars. The same holds for the NLRs of these objects. However, the detection rate of structured radio emission and NLRs drops significantly with redshift, which can most likely be attributed to resolution effects as structures smaller than the beam size are "averaged out". This is also the reason for the higher diversity seen in Seyferts. However, the linear scales in RQQs are up to five times larger than that in Seyfert galaxies, reflecting the more powerful central engine. These arguments are supported by the fact that, at the distances of the quasars, the radio structures of the Seyfert galaxies (in the same morphological group) would only be extended by less than an arcsecond.

The structure of the radio emission can also be found in the emission-line gas where one- or two-sidedness appear to dominate in both regimes ${ }^{4}$. Thus, from these morphological points of view the RQQs can be considered as scaled-up versions of Seyfert galaxies. The power of the central engine and of the radio jet has not increased enough to lead to a different scenario in the more powerful RQQs. The interaction of the radio ejecta with the emission-line gas suggested from the corresponding images is confirmed by spectroscopic studies (e.g. Ferruit et al. 1999; Leipski \& Bennert 2006). Therefore the radio jet might be responsible for shaping the NLR gas distribution. However, dense material can alter the shape of the radio and NLR emission significantly as proposed for e.g. ESO 428-G14 and PG 1119+120. It should be noted that already Quillen et al. (1999) suggested that the morphology of the line-emitting region depends on the distribution of dense ambient media. It is likely that the overall structure is mostly defined by the environmental conditions and the presence of a dense interstellar medium. Thus, it cannot be answered here if the radio jet is responsible for the

${ }^{4}$ While it should be noted that there are only two clear cases for direct radio jet-NLR interaction in our quasar sample, the trends for one- or two-sidedness seem to hold even at higher redshift. overall NLR morphology. Nevertheless the jet is strongly influencing the structure on smaller scales and the velocity field of the emission-line gas.

\subsection{On the size of the NLR and radio emission}

A correlation between the [O III] luminosity and the radio luminosity of quasars has been known for several years (e.g. Miller et al. 1993). This correlation seems to hold, at least for radioquiet objects, if low-luminosity AGN (LLAGN) are added (e.g. Falcke et al. 1995; Xu et al. 1999).

In addition to these results, the close connection of the NLR and radio emission of Seyfert galaxies and radio-quiet quasars found here suggests that also the sizes of these regions may be correlated among radio-quiet objects. Given the availability of the first direct quasar NLR sizes (Bennert et al. 2002, this paper), we can now test such a relation. We increased the database of our sample by additionally including the datasets of Schmitt et al. (2001, 2003) and Kinney et al. (2000), comprising sizes and luminosities of the [O III] and radio emitting regions of several Seyfert galaxies. This sample is homogeneous in terms of data reduction and size determination techniques which were carried out by the same team. The sizes and luminosities were re-calculated according to the world model used here (Sect. 3). We also added PG $1119+120$ for which no direct size measurements of the NLR are available. However, Leipski \& Bennert (2006) have detected [O III] emission associated with the radio knots (Fig. 9). Thus, we estimate that the size of the NLR in PG $1119+120$ is roughly the same as measured for the radio emission.

Combining these data, a correlation between the NLR size and radio size is indeed found (Fig. 12), supporting the connection of both measures. For all objects a linear least square fit yields $R_{\mathrm{NLR}} \propto R_{\text {radio }}^{0.38 \pm 0.05}$. Note that the result of the fit does not change within the errors when all sources with upper limits on either measure are excluded.

Moreover, projection effects should cancel out in this correlation, if one assumes that the axis of the "escaping cone" of ionising photons and the direction of the radio jet is the same. This is supported by results of Schmitt et al. (2003) who find a very good alignment between the position angle (PA) of the extended [O III] emission and that of the radio jet, confirming previous studies (e.g. Wilson \& Tsvetanov 1994).

Given that the NLR size increases with [O III] luminosity as implied by the NLR size-luminosity relation (Bennert et al. 2002) and assuming that the [O III] luminosity can be used as a measure for the total luminosity of the AGN, the radio emitting region becomes larger when the total luminosity is increased. Such a behaviour can also be seen from the combination of the $L_{[\mathrm{O} \text { III }}-L_{\text {radio }}$ correlation (e.g. Miller et al. 1993) and the radio size-luminosity relation for radio-quiet quasars (e.g. Morganti et al. 1999). Thus, a possible interpretation of the observed correlation between NLR and radio size is that both depend on the intrinsic luminosity of the central engine. However, it is not clear whether also the interaction between radio jets and NLR gas influences the correlation.

From Fig. 12, it is noticeable that the radio radius increases faster than the NLR radius when the (total) luminosity of the AGN is powered up. The radio jet is more likely to penetrate deeper into the surrounding medium before being stopped and disrupted (see also Barvainis et al. 2005). The NLR increases by the presence of more high-energy photons of the AGN, that ionise the ambient gas. Thus, tuning up the central luminosity, the size of the NLR grows while the radio jet digs its way 


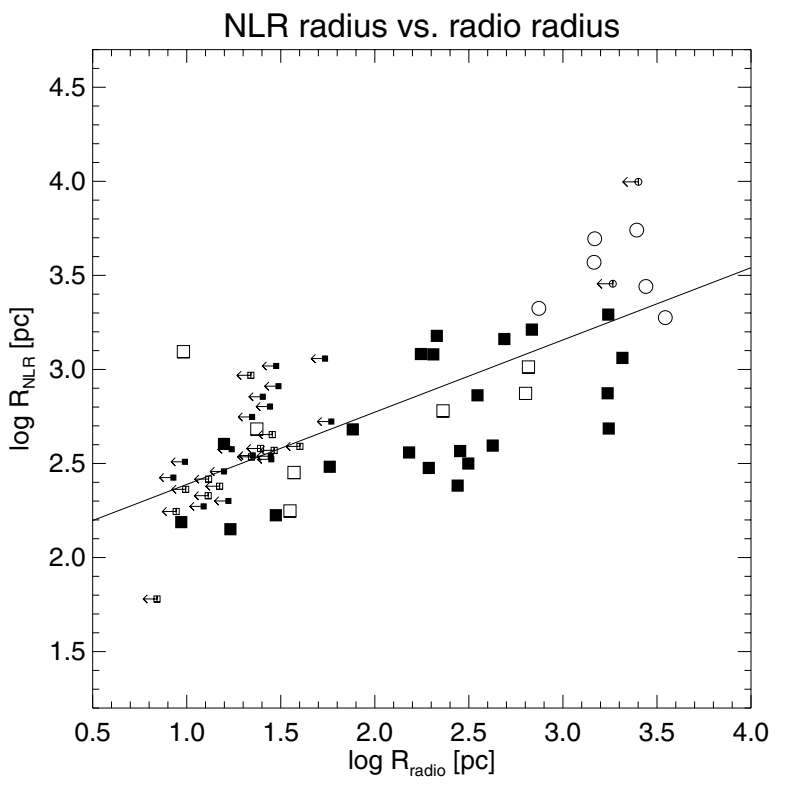

Fig. 12. Correlation of the radii of the [O III] and radio emitting regions. Filled symbols represent type-2, open symbols type-1 objects, arrows denote upper limits. The RQQs data (circles) were taken from Bennert et al. (2002) and from this paper, while the Seyfert data were taken from Falcke et al. (1998), Kinney et al. (2000), and Schmitt et al. (2001, 2003 ). The linear least square fit corresponds to $R_{\mathrm{NLR}} \propto R_{\mathrm{radio}}^{0.38 \pm 0.05}$. Note that a fit excluding data points with upper limits is the same within the errors.

through the interstellar medium to overtake the NLR gas at some point. Only for sufficiently high jet energies the jet is likely to terminate outside the host galaxy (Falcke et al. 1995).

\section{Conclusions}

We present deep radio images of 14 radio-quiet quasars and six Seyfert galaxies that show extended structures which can be interpreted as jets. Especially for the RQQs, aligned multiple components are detected that are generally interpreted as termination points of radio jets. The radio structures of RQQs are larger than those of Seyfert galaxies but exhibit the same morphology. Thus, RQQs can be regarded as scaled-up versions of Seyfert galaxies, keeping in mind distance and resolution effects when interpreting the images. The similarity can also be seen in the NLR gas distribution where the radio jet of the RQQs leaves its imprint on the NLR creating structures that are well known from Seyfert NLRs. This includes sweeping-up material and the creation of bow-shocks (PG 0157+001 vs. Mrk 573) as well as one-sided jets which interact with one-sided NLRs (PG 1012+008 vs. ESO 428-G14).

Comparing our results with (radio) snapshot surveys of Seyfert galaxies and quasars, we have shown that these surveys can miss significant structures. The apparent majority of unresolved RQQs in contrast to the Seyferts in our dataset can be attributed to selection and resolution effects.

Including literature data of Seyfert NLRs and their radio emission, we find a clear correlation between the size of the NLR and the size of the radio emission. We conclude that there is no significant morphological difference between Seyfert galaxies and radio-quiet quasars on scales of typical NLR sizes (hundreds of $\mathrm{pc}$ to $\mathrm{kpc}$ ), neither in the radio nor in the [O III] emitting region. Moreover, the interaction of radio-ejecta with the NLR gas seem to be equally important in both types of sources for shaping the structure of the NLR and radio emission.

Acknowledgements. Part of this work was supported by Sonderforschungsbereich SFB 591 "Universelles Verhalten gleich-gewichtsferner Plasmen" der Deutschen Forschungsgemeinschaft. We also acknowledge the comments of the anonymous referee.

\section{Appendix A: Seyfert galaxies for morphological comparison}

These six objects were selected on the basis of their known extended radio emission. Sources with various morphologies were chosen. They are bright enough to be observed at $8.4 \mathrm{GHz}$ to benefit from the higher angular resolution at this frequency. We use them to compare the structure of the extended radio emission of quasars and Seyfert galaxies. The higher linear resolution of the Seyfert maps may provide clues to the underlying structure in the extended quasar emission that is averaged out. Any gradual transition from Seyferts to quasars with increasing luminosity should conserve the basic morphological features to some extent. As already shown for the 14 RQQs and known from the literature, the long integration times enable us to detect low flux level emission of very diverse structures which is missed in snapshot surveys.

Mrk 612. Four different components can be identified in this source (Fig. A.1, left). Three of them are aligned in north-south direction over $2^{\prime \prime}$, whereas the fourth one lies closely to the west of the brightest source. Nagar et al. (1999) identified the most southern component to be the central one. Since the northern structure is elongated perpendicular to the source axis they interpreted this as a transverse shock.

ESO 428-G14. A prominent, bended, diffuse one-sided jet dominates the structure of this Seyfert-2 galaxy (Fig. A.1, right). From the central component, the jet heads towards the south-east and bends by nearly 90 degrees to the north-east after approximately $4^{\prime \prime}$. With better resolution (but a lack of signal), Falcke et al. (1998) have shown this structure also at $14.9 \mathrm{GHz}$. Our radio map and the emission-line images of Falcke et al. (1996a) demonstrate the striking structural similarity of radio emission and NLR emission (from HST [O III] and $\mathrm{H} \alpha$ images) in this source.

NGC 2639. The brightest source in our sample shows a $2^{\prime \prime}$ wide structure of three components that are merged at the available resolution (Fig. A.2, left). Interestingly, there is some diffuse emission around the two outer components that is constricted at the position of the central source. Wilson et al. (1998) reported a VLBA nucleus and the variability of this source. In fact, their flux on arcsecond scales from an earlier VLA map (Ulvestad \& Wilson 1989) was smaller than their VLBA flux. This flux variablity is also obvious when including our data: Our peak flux is 5 times greater than the flux measured by Ulvestad \& Wilson (1989) and 3 times greater than that of Wilson et al. (1998). Since the measurements in the literature stated above are at $5 \mathrm{GHz}$, they are only comparable with our fluxes at $8.4 \mathrm{GHz}$ due to the very flat spectral index of the central (dominating) source above $5 \mathrm{GHz}$ (Wilson et al. 1998).

NGC 2992. This object shows probably the most interesting structure (Fig. A.2, right). The very prominent $\mathrm{S}$-shaped radio emission is roughly $8^{\prime \prime}$ wide and coincides with a nuclear outflow of emission-line gas (Veilleux et al. 2001). Their model of a hot, bipolar, thermal wind that interacts with the ISM of the galaxy excludes a significant contribution of the radio-jet to the energy budget of the NLR. At lower (radio) frequencies 

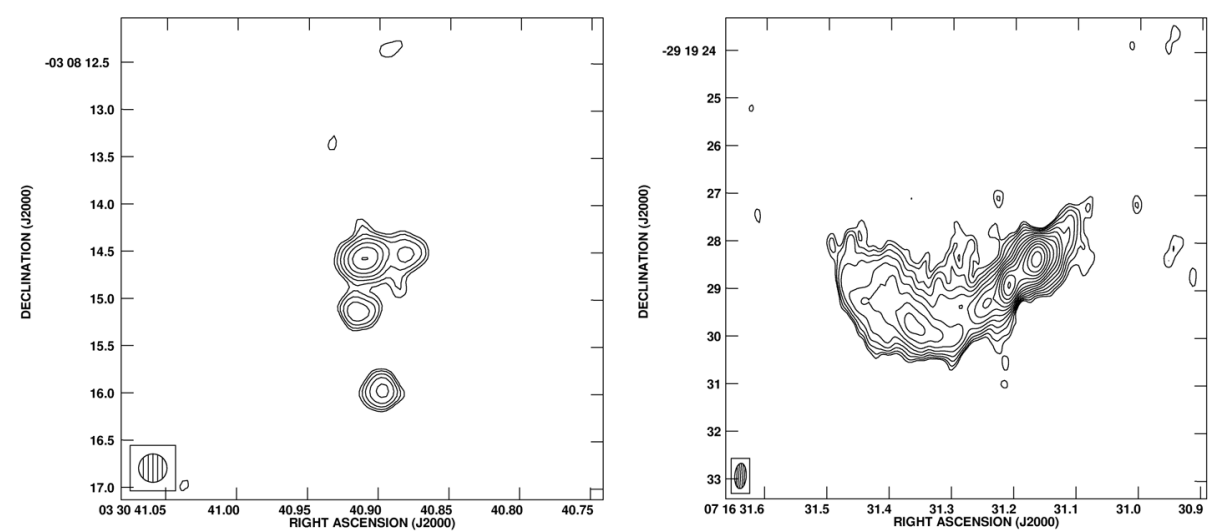

Fig. A.1. VLA images of Mrk 612 (left) and ESO 428-G14 (right). Left: $5^{\prime \prime} \times 5^{\prime \prime}$ wide image. Natural-weighted A-Array map at 8.4 GHz with a $0.3 \times 0$. .'3 beam. Right: $10^{\prime \prime} \times 10^{\prime \prime}$ wide image. Natural-weighted VLA A-Array map at $8.4 \mathrm{GHz}$ with a $0.53 \times 0.0^{\prime} 24$ beam.
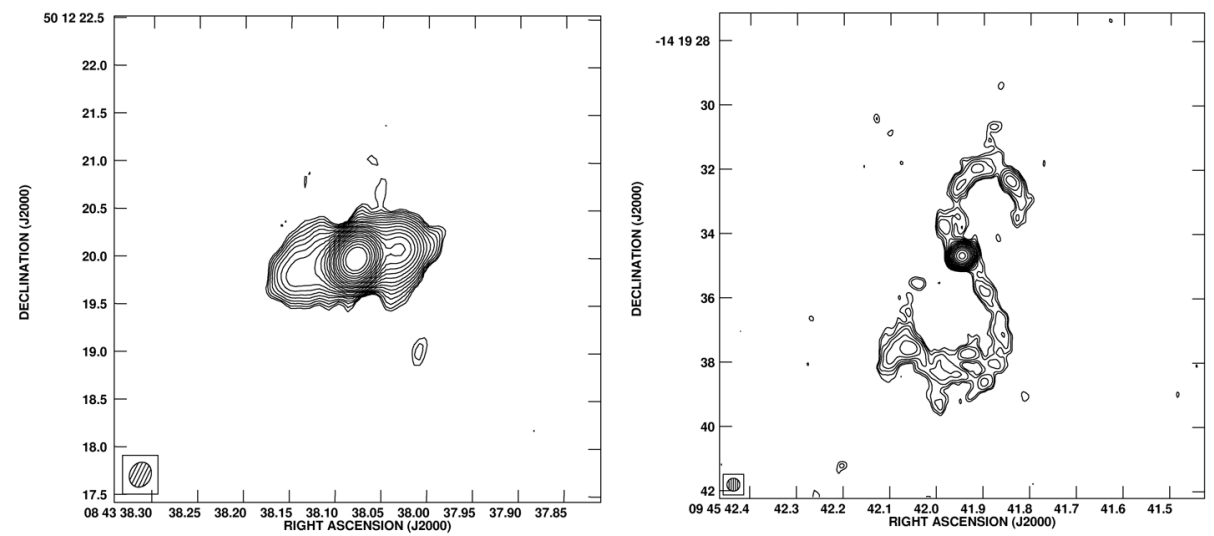

Fig. A.2. VLA images of NGC 2639 (left) and NGC 2992 (right). Left: $5^{\prime \prime} \times 5^{\prime \prime}$ wide image. Natural-weighted A-Array map at 8.4 GHz with a $0.27 \times 0$ ' 22 beam. Right: $15^{\prime \prime} \times 15^{\prime \prime}$ wide image. Natural-weighted VLA A-Array map at $8.4 \mathrm{GHz}$ with a $0{ }^{\prime} 4 \times 0{ }^{\prime} 4$ beam.
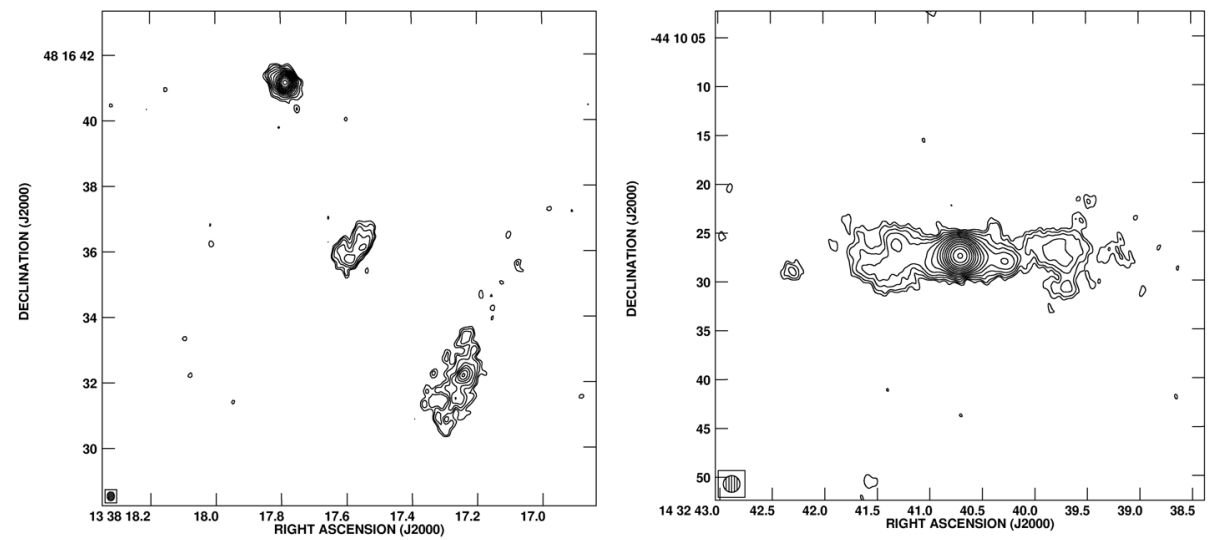

Fig. A.3. VLA images of Mrk 266 (left) and NGC 5643 (right). Left: $15^{\prime \prime} \times 15^{\prime \prime}$ wide image. Natural-weighted A-Array map at 8.4 GHz with a $0.27 \times 0$ ' 22 beam. Right: $20^{\prime \prime} \times 20^{\prime \prime}$ wide image. Natural-weighted VLA A-Array map at $8.4 \mathrm{GHz}$ with a 1'.7 $71^{\prime \prime} 7$ beam.

(5 GHz), the two loops are closed and a figure- 8 structure appears (Ulvestad \& Wilson 1984).

Mrk 266. This source consists of three components: one compact core as well as two diffuse emission regions (Fig. A.3, left). All sources are well aligned and the whole structure is roughly $12^{\prime \prime}$ long. This galaxy with a double-nucleus in the optical shows LINER emission in the north-eastern nucleus whereas the south-western nucleus is a Seyfert-2 type object (Mazzarella et al. 1988). They conclude that this galaxy is a merger of a Seyfert and a LINER galaxy. The north-eastern radio component most likely belongs to the LINER nucleus.
This interpretation is strengthened by the fact that compact radio structures are well known for LINER galaxies (e.g. Nagar et al. 2000). On the other hand, the south-western structure belongs to the Seyfert galaxy. The radio structure of this region with double-sided jet-like diffuse emission is very typical for Seyfert galaxies. The diffuse emission between both components is considered to be the region of interaction with enhanced synchrotron emission.

NGC 5643. This source has a diffuse radio jet on both sides of the nucleus which is nearly $30^{\prime \prime}$ long (Fig. A.3, right). The whole radio structure matches well with the corresponding 
distribution of the NLR gas (Simpson et al. 1997). Although Simpson et al. (1997) detect only a one-sided ionisation cone on the eastern side of the nucleus they state that the western side suffers heavy extinction by a dust lane, thus shadowing the second cone.

\section{References}

Bahcall, J. N., Kirhakos, S., Saxe, D. H., \& Schneider, D. P. 1997, ApJ, 479, 642 Barvainis, R., \& Antonucci, R. 1989, ApJS, 70, 257

Barvainis, R., Lehár, J., Birkinshaw, M., Falcke, H., \& Blundell, K. M. 2005, ApJ, 618, 108

Bennett, C. L., Halpern, M., Hinshaw, G., et al. 2003, ApJS, 148, 1

Bennert, N., Falcke, H., Schulz, H., Wilson, A. S., \& Wills, B. J. 2002, ApJ, 574, L105

Blundell, K. M., \& Beasley, A. J. 1998, MNRAS, 299, 165

Blundell, K. M., \& Rawlings, S. 2001, ApJ, 562, L5

Capetti, A., Axon, D. J., Macchetto, F., Sparks, W. B., \& Boksenberg, A. 1996, ApJ, 469, 554

de Ruiter, H. R., Parma, P., Fanti, C., \& Fanti, R. 1990, A\&A, 227, 351

Falcke, H., \& Biermann, P. L. 1995, A\&A, 293, 665

Falcke, H., Malkan, M. A., \& Biermann, P. L. 1995, A\&A, 298, 375

Falcke, H., Wilson, A. S., Simpson, C., \& Bower, G. A. 1996a, ApJ, 470, L31

Falcke, H., Sherwood, W., \& Patnaik, A. R. 1996b, ApJ, 471, 106

Falcke, H., Wilson, A. S., \& Simpson, C. 1998, ApJ, 502, 199

Fanaroff, B. L., \& Riley, J. M. 1974, MNRAS, 167, 31

Ferruit, P., Wilson, A. S., Falcke, H., et al. 1999, MNRAS, 309, 1

Haas, M., Siebenmorgen, R., \& Schulz, B. 2005, A\&A, 442, L39

Ho, L. C., \& Ulvestad, J. S. 2001, ApJS, 133, 77

Kellermann, K. I., Sramek, R., Schmidt, M., Shaffer, D. B., \& Green, R. 1989, AJ, 98, 1195

Kellermann, K. I., Sramek, R., Schmidt, M., Green, R. F., \& Shaffer, D. B. 1994, AJ, 108, 1163

Kinney, A. L., Schmitt, H. R., Clarke, C. J., et al. 2000, ApJ, 537, 152

Kukula, M. J., Pedlar, A., Baum, S. A., \& O'Dea, C. P. 1995, MNRAS, 276, 1262
Kukula, M. J., Dunlop, J. S., Hughes, D. H., \& Rawlings, S. 1998, MNRAS, 297, 366

Leipski, C., \& Bennert. N. 2006, A\&A, 448, 165

Mazzarella, J. M., Gaume, R. A., Aller, H. D., \& Hughes, P. A. 1988, ApJ, 333, 168

Miller, L., Peacock, J. A., \& Mead, A. R. G. 1990, MNRAS, 244, 207

Miller, P., Rawlings, S., \& Saunders, R. 1993, MNRAS, 263, 425

Morganti, R., Tsvetanov, Z. I., Gallimore, J., \& Allen, M. G. 1999, A\&AS, 137, 457

Nagar, N. M., Wilson, A. S., Mulchaey, J. S., \& Gallimore, J. F. 1999, ApJS, 120,209

Nagar, N. M., Falcke, H., Wilson, A. S., \& Ho, L. C. 2000, ApJ, 542, 186

Nagar, N. M., Falcke, H., \& Wilson, A. S. 2005, A\&A, 435, 521

Neeser, M. J., Eales, S. A., Law-Green, J. D., Leahy, J. P., \& Rawlings, S. 1995, ApJ, 451, 76

Pogge, R. W., \& de Robertis, M. M. 1995, ApJ, 451, 585

Quillen, A. C., Alonso-Herrero, A., Rieke, M. J., et al. 1999, ApJ, 527, 696

Rush, B., Malkan, M. A., \& Edelson, R. A. 1996, ApJ, 473, 130

Schmidt, M., \& Green, R. F. 1983, ApJ, 269, 352

Schmitt, H. R., Ulvestad, J. S., Antonucci, R. R. J., \& Kinney, A. L. 2001, ApJS, 132,199

Schmitt, H. R., Donley, J. L., Antonucci, R. R. J., Hutchings, J. B., \& Kinney, A. L. 2003, ApJS, 148, 327

Simpson, C., Wilson, A. S., Bower, G., et al. 1997, ApJ, 474, 121

Stockton, A., \& MacKenty, J. W. 1987, ApJ, 316, 584

Thompson, A. R., Clark, B. G., Wade, C. M., \& Napier, P. J. 1980, ApJS, 44, 151

Ulvestad, J. S., \& Wilson, A. S. 1984, ApJ, 285, 439

Ulvestad, J. S., \& Wilson, A. S. 1989, ApJ, 343, 659

Ulvestad, J. S., \& Ho, L. C. 2001, ApJ, 558, 561

Ulvestad, J. S., Wilson, A. S., \& Sramek, R. A. 1981, ApJ, 247, 419

Ulvestad, J. S., Antonucci, R. R. J., \& Barvainis, R. 2005, ApJ, 621, 123

Veilleux, S., Shopbell, P. L., \& Miller, S. T. 2001, AJ, 121, 198

Wampler, E. J., \& Ponz, D. 1985, ApJ, 298, 448

Wilson, A. S., \& Tsvetanov, Z. I. 1994, AJ, 107, 1227

Wilson, A. S., Roy, A. L., Ulvestad, J. S., et al. 1998, ApJ, 505, 587

Xu, C., Livio, M., \& Baum, S. 1999, AJ, 118, 1169 